\title{
Actitud hacia la matemática: percepción de la actitud de padres
}

Por: Dr. Luis Gerardo Meza-Cascante, Instituto Tecnológico de Costa Rica, Costa Rica ${ }^{1}$, Dra. Evelyn Agüero-Calvo, Instituto Tecnológico de Costa Rica, Costa Rica², Dra. Zuleyka Suárez-Valdés-Ayala, Instituto Tecnológico de Costa Rica, Costa Rica ${ }^{3}$, Dra. Martha Calderón-Ferrey, Instituto Tecnológico de Costa Rica, Costa Rica ${ }^{4}$, Mag. Laura SanchoMartínez, Instituto Tecnológico de Costa Rica, Costa Rica ${ }^{5}$, Dra. Patricia Pérez-Tyteca, Universidad de Alicante, España ${ }^{6}$ y Dr. Javier MonjeParrilla, Universidad de Alicante, España ${ }^{7}$

Recibido: $\quad 12$ de febrero, 2019.

Aceptado: $\quad \mathbf{1}^{\mathrm{o}}$ de abril, 2019.

1 El Prof. Luis Gerardo Meza es Doctor en Educación, graduado en la Universidad Estatal a Distancia de Costa Rica. Labora en la Escuela de Matemática del Instituto Tecnológico de Costa Rica, como docente Catedrático e Investigador consolidado 1. Contacto: gemeza@ tec.ac.cr.

2 La Doctora Evelyn Agüero es Doctora en Intervención Educativa por la Universidad de Valencia en España. Labora en la Escuela de Matemática del Instituto Tecnológico de Costa Rica como docente Catedrática e Investigadora consolidada 1. Contacto: evaguero@tec. ac.cr. Contacto: evaguero@tec.ac.cr.

3 La Dra. Zuleyka Suárez-Valdés-Ayala obtuvo su doctorado en Educación en la Universidad Estatal a Distancia de Costa Rica. Labora como docente Catedrática-Intermedio e Investigadora consolidada 1 en la Escuela de Matemática del Instituto Tecnológico de Costa Rica. Contacto: zsuarez@tec.ac.cr.

$4 \quad$ La Dra. Martha Calderón Ferrey se graduó como Doctora en Derecho por la Universidad Estatal a Distancia de Costa Rica, y también como Doctora en Intervención Educativa por la Universidad de Valencia, España. Labora como docente e investigadora en la Escuela de Ciencias Sociales del Instituto Tecnológico de Costa Rica. Contacto: micalderon@tec. ac.cr.

5 La Mag. Laura Sancho Martínez obtuvo su maestría en Danza, en la Universidad Nacional de Costa Rica. Es docente e investigadora de la Escuela de Cultura y Deporte del Instituto Tecnológico de Costa Rica. Contacto: lasancho@tec.ac.cr.

6 La Dra. Patricia Pérez-Tyteca es Doctora en Didáctica de la Matemática y trabaja en el departamento de Innovación y Formación Didáctica, de la Universidad de Alicante, España. Contacto: patricia.perez@ua.es.

7 El Dr. Javier Monje-Parrilla es Doctor en Didácticas específicas en la especialidad de didáctica de la Matemática. Se desempeña en el departamento de Innovación y Formación Didáctica de la Universidad de Alicante, España. Contacto: monjejavier@ua.es.

PALABRAS CLAVE:

actitud, matemática, educación secundaria.

\section{KEY WORDS:}

attitude, math, secondary education. 


\section{Resumen}

Seguidamente, se presentan los resultados de un estudio en educación matemática, del dominio afectivo, sobre la relación entre las variables "actitud hacia la matemática", "percepción de la actitud hacia la matemática del padre" y "percepción de la actitud hacia la matemática de la madre", en estudiantes de la educación media costarricense, realizado en el 2017. Se concluye que no se encuentran diferencias en la primera variable por sexo, pero sí en las otras dos, con niveles superiores para los varones y tamaño del efecto pequeño. Además, se detectan relaciones significativas, positivas y moderadas entre las tres variables.

\section{Attitude towards mathematics: perception of the attitude of parents}

\section{Abstract}

This research presents the results of a study in mathematics education on affective domain analyzing the relationship between three variables: "attitude towards mathematics," "perception of the attitude toward mathematics," and "the student's mother's perceptions of the attitude toward mathematics" among Costa Rican secondary students in 2017. The study concludes that there are no differences on the first variables according to gender, yet the other two show higher levels for males and small effect size. Furthermore, significant relationships, both positive and moderate, are found among all three variables.

\section{INTRODUCCIÓN}

Las investigaciones que contemplan el componente afectivo se enmarcan dentro del denominado "dominio afectivo", entendido a partir de McLeod (1989, p. 245), uno de los pioneros en el campo, como "un extenso rango de estados de ánimo que son generalmente considerados como algo diferente de la pura cognición" e incluye como componentes específicos las creencias, las actitudes y las emociones.

En los procesos de enseñanza y de aprendizaje de esta disciplina, suelen encontrarse numerosos grupos de estudiantes que la conciben de manera negativa y que, además, asumen el conocimiento matemático como complejo y generador de sentimientos de intranquilidad, ansiedad, miedo, inseguridad, desconcierto e incertidumbre (Gil, Blanco y Guerrero, 2006; Martínez, 2008). Un estudio de Meza y Azofeifa (2009) evidenció que, para el caso costarricense, los estudiantes muestran una actitud negativa hacia la matemática y la consideran una disciplina aburrida, confusa, frustrante, estresante y compleja.

Dado que ese panorama no es nuevo, se han desarrollado investigaciones desde hace muchos años en procura de conocimiento que permita atender la situación, inicialmente con énfasis predominante en lo cognitivo (Gil, Blanco y Guerrero, 2006; Gómez-Chacón, 2010), con creciente incorporación de aspectos afectivos en los últimos años (Gómez-Chacón, 2010, Pérez-Tyteca, 2012), esto debido a que "distintos investigadores han puesto de manifiesto que los afectos (emociones, actitudes y creencias) de los estudiantes son factores claves en la comprensión de su comportamiento en matemáticas" (Gil, Blanco y Guerrero, 2006, p. 17).

Las creencias, actitudes y emociones son factores importantes en el estudio del dominio afectivo en 
la educación matemática, y varían tanto en nivel de intensidad como de estabilidad. Por ejemplo, "las creencias y actitudes son relativamente estables y resistentes al cambio, pero las respuestas emocionales pueden cambiar rápidamente" (McLeod, 1989, p. 246).

La importancia de estudiar el aspecto afectivo está, de acuerdo con Gómez-Chacón (2000), en que la relación entre los afectos (emociones, actitudes y creencias) y el rendimiento académico es cíclica: la experiencia que tiene el estudiante al aprender matemática le provoca distintas reacciones que influyen en la formación de sus creencias, pero a la vez, las creencias del sujeto tienen una consecuencia directa en su comportamiento en situaciones de aprendizaje y en su capacidad para aprender.

Las creencias parecen tener influencia en los estudiantes ya que, por un lado, están las creencias acerca de la matemática como disciplina que forman parte del contexto y, por otro lado, están las creencias acerca de sí mismos y su relación con la matemática, las cuales tienen un fuerte componente afectivo relacionado con los éxitos y fracasos (McLeod, 1989).

Las emociones de los estudiantes no han sido objeto de mayor estudio en las investigaciones debido que se buscan factores que sean estables y medibles mediante un cuestionario, aunque algunos investigadores han detectado que las emociones influyen en los procesos cognitivos. Las reacciones emocionales se pueden describir como miedo, ansiedad, vergüenza o pánico (McLeod, 1989).

Entre el espectro de variables que integran el dominio afectivo, destaca la actitud hacia la matemática. "Las actitudes son aquellas que expresan algún grado de aprobación o desaprobación, gusto o disgusto, acercamiento o alejamiento" (Mato, 2006, p. 40). Pérez-Tyteca, Castro, Rico y Castro (2011, p. 238) entienden la actitud hacia la matemática como "la predisposición aprendida de los estudiantes a responder de manera positiva o negativa a las matemáticas, lo que determina su intención e influye en su comportamiento ante la materia". Se puede pensar en las actitudes como el resultado final de una reacción emocional que se ha automatizado.
Por ejemplo, "los estudiantes que son temerosos en ciertos entornos matemáticos pueden eventualmente volverse ansiosos crónicos, pero si un estudiante tiene regularmente experiencias positivas con problemas matemáticos no rutinarios, entonces podría desarrollar una actitud de curiosidad y entusiasmo hacia la resolución de problemas" (McLeod, 1989, p.249).

Gómez-Chacón (2000) clasifica las actitudes en dos grandes clases: actitudes hacia las matemáticas y actitudes matemáticas. En la primera categoría incluye aspectos relacionados con la valoración y el aprecio de la matemática como disciplina, al interés por esta materia y por su aprendizaje. Esta perspectiva es la que se utilizó en la investigación, por cuanto el instrumento de medición utilizado responde a este perfil.

La relevancia de estudiar la actitud hacia la matemática estriba, de acuerdo con Akay y Boz (2010), en que la mayoría de las investigaciones que la abordan revelan una relación positiva entre la actitud hacia la matemática y el éxito académico. Las investigaciones de Aparicio y Bazán (2005), Auzmendi (1992), Carmona (2004) y Gil (1999), reseñadas por Montero, Pedroza, Astiz y Vilanova (2015), apoyan esa tesis pues han encontrado correlación positiva entre la actitud hacia la matemática de los estudiantes y su rendimiento en esta materia.

La investigación extiende a nivel país los resultados previos obtenidos por Sanabria (2016) de una muestra localizada en un contexto geográfico pequeño.

\section{REFERENTES CONCEPTUALES}

Desde hace varios años se han realizado estudios sobre la incidencia de la familia en los procesos educativos formales, en general, y sobre la enseñanza y el aprendizaje de la matemática, en particular. Banks (1964, citado en Quiles, 1993, p. 116) afirma que "las actitudes matemáticas de los padres y profesores explican buena parte de la varianza de las actitudes de los alumnos hacia la asignatura".

El informe Crockroft (1985, citado en Gairín, 1990) daba cuenta de que la participación de los padres, incluso de modo inconsciente, puede 
ejercer considerable influencia sobre la actitud de sus hijos ante la matemática. En otro estudio atribuido a Poffenberger y Norton (1959, citados en Gairín, 1990), se señalaba que las actitudes del hogar, entre otras variables, desempeñaban papeles fundamentales en la determinación de las actitudes hacia la matemática.

A lo anterior, es necesario agregar los aportes de estudios más recientes, los cuales han evidenciado que un buen predictor de éxito escolar está en las expectativas de los padres sobre los logros académicos de sus hijos (Michigan Department of Education, 2001 y Epstein, 2013, citados en Romagnoli y Cortese, 2015).

Mato, Muñoz y Chao (2014) han reseñado investigaciones de Besharat (2003), Peleg-Popko (2002), González-Pienda, Núñez, González-Pumariega, Álvarez, Roces y García (2003), Magalhães (2007) y Torío, Peña-Calvo e Inda-Caro (2008), que muestran que el papel que desempeñan los padres en la dimensión afectiva de los hijos respecto a la matemática es determinante en el proceso de enseñanza-aprendizaje de la disciplina.

En el ámbito costarricense, se conocen los estudios en la educación secundaria de Sanabria (2016) y Leonhardes (2017) sobre las variables "percepción de la actitud hacia la matemática de la madre" y "percepción de la actitud hacia la matemática del padre".

Sanabria (2016) por su parte, estudió la relación entre la "actitud hacia la matemática", la "percepción de la actitud de la madre hacia la matemática" y la "percepción de la actitud del padre hacia la matemática", en tres colegios públicos de la provincia de Cartago en el año 2015, y halló relaciones significativas, entre ellas, las siguientes: moderada $(r=0.312)$ entre la primera y la segunda, débil $(r=0.262)$ entre la primera y la tercera y moderada $(r=0.666)$ entre las dos últimas. Además, encontró que la "percepción de la actitud hacia la matemática de la madre" aporta más a explicar la varianza de la variable "actitud hacia la matemática" (beta=0.248) que la "percepción de la actitud hacia la matemática del padre" (beta=0.097).
Leonhardes (2017) estudió también las variables "percepción de la actitud de la madre hacia la matemática" y "percepción de la actitud del padre hacia la matemática" y su relación con "la expectativa de éxito en matemática", también en tres colegios públicos de la provincia de Cartago, en 2016. Los resultados revelan una relación positiva, significativa y moderada entre las dos primeras $(r=0.628)$, entre la primera y la tercera $(r=0.459)$ y entre la segunda y la tercera $(r=0.424)$.

Sanabria (2016), adicionalmente, detectó diferencias en promedio en el nivel de percepción de la actitud hacia la matemática de la madre entre hombres y mujeres, mas no en el caso de la variable percepción de la actitud hacia la matemática del padre, mientras que Leonhardes (2017) encontró diferencias según el sexo de los estudiantes para ambas variables.

\section{METOdOLOGÍA}

La investigación es de tipo cuantitativo y dentro de este enfoque se clasifica como descriptiva y correlacional.

\section{Participantes}

El sistema educativo en Costa Rica se divide en cuatro niveles: preescolar, primaria, secundaria y superior. La educación secundaria abarca los niveles de sétimo, octavo y noveno y una fase de educación diversificada.

La muestra estuvo integrada por 3100 estudiantes de sétimo a undécimo año de colegios públicos diurnos oficiales, del Ministerio de Educación Pública de Costa Rica, matriculados en 2017, como se muestra en las tablas 1 y 2 :

Tabla 1

\section{Distribución por sexo}

\begin{tabular}{|c|c|c|}
\hline Sexo & Frecuencia & Porcentaje \\
\hline Hombre & 1600 & 51.6 \\
\hline Mujer & 1500 & 48.4 \\
\hline
\end{tabular}

Fuente: Elaboración propia 
Tabla 2

Distribución por nivel educativo

\begin{tabular}{|l|l|l|}
\hline Nivel & Frecuencia & Porcentaje \\
\hline 7 & 639 & 20.61 \\
\hline 8 & 616 & 19.87 \\
\hline 9 & 638 & 20.58 \\
\hline 10 & 583 & 18.81 \\
\hline 11 & 624 & 20.13 \\
\hline
\end{tabular}

Fuente: Elaboración propia

La selección de los colegios participantes se realizó por muestreo simple aleatorio estratificado, según la zona de ubicación (Tabla 3), y de acuerdo con la población estudiantil de cada provincia.

\section{Tabla 3 \\ Distribución según tipo de región de ubicación del colegio}

\begin{tabular}{|l|l|l|}
\hline Tipo de colegio & Frecuencia & Porcentaje \\
\hline Urbano & 2083 & 67.2 \\
\hline Rural & 1017 & 32.8 \\
\hline \multicolumn{2}{|c|}{ Fuente: Elaboración propia }
\end{tabular}

\section{Instrumentos de medición}

La actitud hacia la matemática, la percepción de la actitud hacia la matemática de la madre y la percepción de la actitud hacia la matemática del padre, también fueron medidas utilizando las correspondientes subescalas de la escala de actitud hacia la matemática de Fennema y Sherman (1976), consistentes cada una en 12 ítems tipo Likert con cinco opciones desde "totalmente de acuerdo" a "totalmente en desacuerdo", las cuales han sido validadas durante más de 30 años en diversas investigaciones (Nortes y Nortes, 2014; Berenguel, Gil, Montoro y Moreno, 2015), e indican que, aunque las escalas forman parte de un cuestionario sobre actitudes, pueden usarse por separado. Con las tres subescalas utilizadas, se integró un instrumento de 36 ítems autoadministrado, que cada estudiante respondió de manera anónima y confidencial (véase el Anexo).

\section{Procedimiento}

Se aplicó el instrumento al segundo grupo de cada nivel en cada colegio seleccionado, con una intro- ducción previa seguida de las instrucciones correspondientes. Las respuestas fueron recolectadas en un formato de lápiz y papel en lapsos aproximados de 15 minutos por grupo.

\section{Análisis estadístico}

Para cada una de las subescalas se realizó un estudio de sus características psicométricas: índices de discriminación de los ítems, unidimensionalidad y confiabilidad; además se clasificaron los puntajes en cinco categorías: muy desfavorable, favorable, neutra, favorable y muy favorable.

Para estudiar la validez de las subescalas se realizó un estudio del índice de discriminación de cada ítem, calculando la correlación entre la puntuación aportada por el ítem y la obtenida por la suma de los otros once ítems (Lozano y De La Fuente-Solana, 2009). La interpretación del valor de los índices de discriminación se realizó con base en las recomendaciones de Lozano y De La Fuente-Solana (2009).

Para la escala de "Actitud hacia la matemática" se obtuvo que todos los ítems alcanzan un valor mínimo aceptable de 0.3 (Lozano y De La Fuente-Solana, 2009), y para la escala de "Percepción de la actitud hacia la matemática de la madre" también todos los ítems obtienen el valor mínimo aceptable. De forma similar, para la escala de "Percepción de la actitud hacia la matemática del padre" los ítems 31 y 36 no alcanzan ese valor mínimo, razón por la cual fueron descartados del análisis.

También se analizó la unidimensionalidad de cada subescala, con la finalidad de evidenciar que se mide fundamentalmente un solo constructo en cada caso, aplicando el análisis factorial por ser la técnica más utilizada para estos efectos (Jiménez y Montero, 2013), previo cálculo del índice de adecuación muestral de Kaiser-Meyer y Olkin (KMO) y de esfericidad de Bartlett. De igual manera, se utilizó el criterio de unidimensionalidad recomendado por Carmines y Zeller (1979, citados en Burga, 2006) que plantea que el primer factor explique al menos el $40 \%$ de la varianza.

De esta manera, se estableció que las tres subescalas muestran evidencia de unidimensionalidad, con valores del índice $\mathrm{KMO}$ de 0.894, 0.829 y 0.826 
respectivamente, con un valor $p<0.05$ y un primer factor que representa más del $40 \%$ de la varianza total explicada en los tres casos, previa eliminación de los ítems 7, 9 y 11 de la escala de "actitud hacia la matemática" y los ítems 13, 14 y 21 de la escala de "percepción de la actitud hacia la matemática de la madre" para lograr que los primeros factores explicaran al menos el $40 \%$ de la varianza.

La confiabilidad (o fiabilidad de la escala) se estudió aplicando la técnica del alfa de Cronbach, siguiendo el planteamiento de Cea (1999) de que un valor mayor o igual a 0.80 es aceptable. Por su parte, el valor del estadístico Alfa de Cronbach fue de 0.855, 0.825 y 0.872 para las subescalas "Actitud hacia la matemática", "Percepción de la actitud hacia la matemática de la madre" y "Percepción de la actitud hacia la matemática del padre", respectivamente, lo cual indica que las tres son adecuadamente confiables.

El análisis estadístico descriptivo de los datos incluye una baremación de los puntajes de cada subescala siguiendo la interpretación que propone PérezTyteca (2012): identificar valores cercanos a 1 con un nivel muy desfavorable de actitud la matemática, valores en torno a 2 con un nivel desfavorable, valores que rondan el 3 como neutrales, los próximos a 4 con un nivel favorable y los valores situados alrededor de 5 con un nivel muy favorable de actitud la matemática. De manera similar se trató a las otras dos variables.

Posteriormente, se contrastaron las siguientes hipótesis:

- Hipótesis 1: No existen diferencias en la actitud hacia la matemática por sexo.

- Hipótesis 2: No existen diferencias en la percepción de la actitud hacia la matemática de la madre por sexo.

- Hipótesis 3: No existen diferencias en la percepción de la actitud hacia la matemática del padre por sexo.

- Hipótesis 4: No existen diferencias en la actitud hacia la matemática por tipo de región donde se ubica el colegio.
- Hipótesis 5: No existen diferencias en la percepción de la actitud hacia la matemática de la madre por tipo de región donde se ubica el colegio.

- Hipótesis 6: No existen diferencias en la percepción de la actitud hacia la matemática del padre por tipo de región donde se ubica el colegio.

- Hipótesis 7: No existe relación entre la actitud hacia la matemática y la percepción de la actitud hacia la matemática de la madre.

- Hipótesis 8: No existe relación entre la actitud hacia la matemática y la percepción de la actitud hacia la matemática del padre.

- Hipótesis 9: No existe relación entre la percepción de la actitud hacia la matemática de la madre y la percepción de la actitud hacia la matemática del padre.

Para el caso de las hipótesis que involucran dos categorías (sexo y zona de ubicación del colegio) se aplica la prueba paramétrica t de Student, asumiendo la normalidad de la distribución de los datos con base en el teorema del límite central. Para las hipótesis que involucran el nivel educativo se emplea la prueba de Welch complementada con la prueba a posteriori de Scheffé. En el caso de las hipótesis sobre la relación entre dos variables se utiliza el coeficiente de correlación de Pearson, previo estudio de la hipótesis de que tal coeficiente pueda ser nulo.

Para los casos con diferencias estadísticamente significativas se calcula el tamaño del efecto utilizando la d de Cohen (Ripoll, 2011), que estima el número de desviaciones típicas que separan a dos grupos. La interpretación de la magnitud de los tamaños del efecto medidos por la d de Cohen se realiza a partir de las referencias dadas por Cohen (Morales, 2008 y Ripoll, 2011): en torno a 0.20 (diferencia pequeña), en torno a 0.50 (diferencia moderada) y 0.80 o más (diferencia grande). 


\section{RESULTADOS}

1. Clasificación del nivel de actitud hacia la matemática, de percepción de la actitud hacia la matemática de la madre y de percepción de actitud de la matemática del padre

El nivel medio de actitud hacia la matemática $(M=34.29, S D=5.98)$ fue significativamente mayor que el promedio de la escala $(t(3100)=67.82$, $p<0.05)$, lo que indica que globalmente los y las estudiantes de la muestra presentan un nivel de actitud hacia la matemática superior al promedio; es decir, manifiestan en general niveles de actitud hacia la matemática favorables. El nivel medio de percepción de la actitud hacia la matemática de la madre $(M=33.31, S D=6.00)$ fue significativamente mayor que el promedio de la escala $(t(3100)=58.53$, $p<0.05)$, lo que indica que globalmente los estudiantes de la muestra presentan un nivel de percepción de la actitud hacia la matemática de la madre superior al promedio; es decir, manifiestan -en general- niveles favorables de percepción de la actitud hacia la matemática de la madre. Por su lado, el nivel medio de percepción de la actitud hacia la matemática del padre $(M=37.54, S D=7.48)$ fue significativamente mayor que el promedio de la escala $(t(3100)=56.09, p<0.05)$, lo que indica que globalmente el estudiantado de la muestra presenta un nivel de percepción de la actitud hacia la matemática del padre superior al promedio; en otras palabras, en general, manifiestan niveles de percepción de la actitud hacia la matemática del padre favorables.

Con respecto a los puntajes obtenidos para la actitud hacia la matemática, percepción de la actitud hacia la matemática de la madre y percepción de la actitud hacia la matemática del padre, en las tablas 4, 5 y 6 se muestran los porcentajes según la categoría:

Tabla 4

\section{Clasificación de actitud hacia la matemática}

\begin{tabular}{|l|l|l|}
\hline Categorías & Frecuencia & Porcentaje \\
\hline Muy desfavorable & 9 & 0.3 \\
\hline Desfavorable & 110 & 3.5 \\
\hline Neutra & 804 & 25.9 \\
\hline Favorable & 1710 & 55.2 \\
\hline Muy favorable & 467 & 15.1 \\
\hline
\end{tabular}

Fuente: Elaboración propia
Tabla 5

\section{Clasificación de percepción de la actitud} hacia la matemática de la madre

\begin{tabular}{|l|l|l|}
\hline Categorías & Frecuencia & Porcentaje \\
\hline Muy desfavorable & 7 & 0.2 \\
\hline Desfavorable & 105 & 3.4 \\
\hline Neutra & 1053 & 34.0 \\
\hline Favorable & 1534 & 49.5 \\
\hline Muy favorable & 401 & 12.9 \\
\hline \multicolumn{2}{|c|}{ Fuente: Elaboración propia }
\end{tabular}

Tabla 6

Clasificación de percepción de la actitud hacia la matemática del padre

\begin{tabular}{|l|l|l|}
\hline Categorías & Frecuencia & Porcentaje \\
\hline Muy desfavorable & 20 & 0.6 \\
\hline Desfavorable & 119 & 3.8 \\
\hline Neutra & 1103 & 35.6 \\
\hline Favorable & 1315 & 42.4 \\
\hline Muy favorable & 543 & 17.5 \\
\hline
\end{tabular}

Fuente: Elaboración propia

Estos resultados señalan que, aproximadamente, un $81,1 \%, 83,5 \%$ y $78 \%$ de las y los estudiantes muestra niveles de actitud hacia la matemática, percepción de la actitud hacia la matemática de la madre y percepción de la actitud hacia la matemática del padre entre favorables y neutros respectivamente.

\section{Contraste de la hipótesis 1}

Para el contraste de la primera hipótesis, se compararon los valores de la media de los hombres $(M=34.23, S D=5.97)$ con los de la media de las mujeres $(M=34.35, S D=6.00)$, y no se encontró una diferencia estadísticamente significativa $(t(3100)=0.577, p>0.05)$. Es decir, se acepta la hipótesis nula y se acoge que no existen diferencias en el nivel de actitud hacia la matemática entre hombres y mujeres.

\section{Contraste de la hipótesis 2}

Para aplicar este procedimiento a la segunda hipótesis, se compararon los valores de la media de los hombres $(M=33.6, S D=5.89)$ con la media de las mujeres $(M=33.01, S D=6.11)$, y se encontró una diferencia estadísticamente significativa con 
tamaño del efecto pequeño $(t(3100)=-2.752$, $p<0.05, \mathrm{~d}=0.099$ ). Es decir, se rechaza la hipótesis nula y se acepta que existen diferencias en el nivel de percepción de la actitud hacia la matemática de la madre entre hombres y mujeres, con niveles mayores en promedio para los hombres.

\section{Contraste de la hipótesis 3}

Para el cotejo de la tercera hipótesis, se compararon los valores de la media de los hombres $(M=37.86$, $S D=7.33)$ con los de las mujeres $(M=37.19$, $S D=7.63)$, y se encontró una diferencia estadísticamente significativa con tamaño del efecto pequeño $(t(3100)=-2.497, p<0.05, d=0.0897)$. En otros términos, se rechaza la hipótesis nula y se admite que hay diferencias en el nivel de percepción de la actitud hacia la matemática del padre entre hombres y mujeres, al punto que los hombres muestran, en promedio, mayores niveles.

\section{Contraste de la hipótesis 4}

Para el contraste de la cuarta hipótesis, se confrontaron los valores de la media de los colegios de la zona urbana $(M=34.00, S D=6.02)$ con las instituciones de la zona rural $(M=34.87, S D=5.86)$, y se encontró una diferencia estadísticamente significativa entre ellos, en relación con el tamaño del efecto pequeño $(t(3100)=-3.788, p<0.05, d=0.145)$. Así las cosas, se rechaza la hipótesis nula y se acepta que existen diferencias en el nivel de actitud hacia la matemática según la zona de ubicación del colegio, con media superior para los estudiantes de los centros educativos ubicados en la zona rural.

\section{Contraste de la hipótesis 5}

Para contrastar la quinta hipótesis, se compararon los valores de la media de los colegios de la zona urbana $(M=33.16, S D=5.95)$ con los de centros educativos de la zona rural $(M=33.61, S D=6.10), y$ no se encontró diferencia estadísticamente significativa entre ellas $(\mathrm{t}(3100)=-1.957, \mathrm{p}>0.05)$. Es decir, se acepta la hipótesis nula y se acoge que no existen diferencias en el nivel de percepción de la actitud hacia la matemática de la madre según la zona de ubicación del colegio.

\section{Contraste de la hipótesis 6}

Con el fin de contrastar la sexta hipótesis, se compararon los valores de la media de las instituciones educativas de la zona urbana $(M=37.33, S D=7.47)$ con la media de los colegios de la zona rural $(M=37.97$, $S D=7.49$ ), y se encontró una diferencia estadísticamente significativa entre ellas con tamaño del efecto pequeño $(t(3100)=-1.957, p>0.05, d=0.085)$. Por ende, se rechaza la hipótesis nula y se asiente que hay diferencias en el nivel de percepción de la actitud hacia la matemática del padre según la zona de ubicación del colegio, con nivel promedio mayor para los estudiantes de la zona rural.

\section{Contraste de la hipótesis 7}

Para contrastar la hipótesis 7, se realizó una correlación de Pearson con resultados de asociación significativa $(r=0.567, p<0.05)$. Así, se rechaza la hipótesis nula y se acepta que existe relación significativa, positiva y moderada entre las variables actitud hacia la matemática y percepción de la actitud hacia la matemática de la madre.

\section{Contraste de la hipótesis 8}

Para contrastar la hipótesis 8, se realizó una correlación de Pearson con resultados de asociación significativa $(r=0.494, p<0.05)$. En otras palabras, se rechaza la hipótesis nula y se reconoce la existencia de una relación significativa, positiva y moderada entre las variables actitud hacia la matemática y percepción de la actitud hacia la matemática del padre.

\section{Contraste de la hipótesis 9}

Para contrastar la hipótesis 9, se realizó una correlación de Pearson con resultados de asociación significativa $(r=0.605, p<0.05)$. Esto significa que se rechaza la hipótesis nula y se admite que existe relación significativa, positiva y moderada entre las variables percepción de la actitud hacia la matemática de la madre y percepción de la actitud hacia la matemática del padre. 


\section{DISCUSIÓN DE RESULTADOS}

La investigación se enfocó en tres variables del dominio afectivo, a saber: actitud hacia la matemática, percepción de la actitud hacia la matemática de la madre y percepción de la actitud hacia la matemática del padre en la educación secundaria oficial diurna costarricense, con el objetivo general de estudiar la relación entre estas variables y la existencia de diferencias en los niveles mostrados por sexo, nivel educativo o zona de ubicación del colegio.

Una cantidad importante de los estudiantes (81,1\%) tiene una actitud hacia la matemática entre favorable y neutra lo que constituye, en principio, un hallazgo positivo. En efecto, de acuerdo con Auzmendi (1992) resulta más fácil aprender aquello que se considera agradable. Aunque los resultados en matemática en la educación media costarricense no alcanzan niveles de rendimiento académico tan altos como los que manifiestan los estudiantes en su actitud hacia la matemática, lo cual no concuerda con las correlaciones positivas entre actitud y rendimiento académico de las investigaciones reseñadas por Montero et al. (2015), se puede considerar, desde una perspectiva optimista y práctica del hallazgo, que puede constituir una base para los procesos de intervención en procura de mejorar el desempeño académico en matemática en la educación media.

De igual modo, los niveles de percepción positiva de los estudiantes de la actitud hacia la matemática de la madre y del padre $(83,5 \%$ y $78 \%$ entre favorable y neutro, respectivamente), es también un resultado positivo, que sugiere una posible agenda de trabajo enfocada en fortalecer la actitud positiva de las madres y de los padres de familia sobre la matemática, su importancia y su utilidad, con el propósito de mantener y fortalecer los niveles detectados de percepción reseñados. Aunque el hallazgo no proviene de una medición directa de la actitud de las madres y de los padres hacia la matemática, sí podría reflejar en alguna medida la realidad que viven sus hijos . Estos resultados reafirman el planteamiento de Banks (1964, citado en Quiles, 1993) sobre la relación positiva entre las actitudes de los padres hacia la matemática y la varianza de las actitudes de las y los estudiantes hacia la matemática.
Por otro lado, no se encontraron diferencias en la actitud hacia la matemática entre hombres y mujeres, resultado que difiere de los obtenidos en otros estudios sobre la actitud hacia la matemática y que son reseñados por Ursini (2014), lo que no resulta del todo inusual como plantea esta misma autora, pero sí entre el nivel de percepción de la actitud hacia la matemática tanto de la madre como del padre, con niveles en promedio ligeramente superiores para los varones, y con tamaño del efecto pequeño en los dos casos. Este resultado no coincide con los obtenidos por Sanabria (2016) o Leonhardes (2017) quienes no encontraron diferencias por sexo para ninguna de esas dos variables, en el caso de muestras menores de estudiantes costarricenses tomadas solo en la provincia de Cartago. Las posibles explicaciones de estas discrepancias las apunta Fennema (1996, citada en Ursini, 2014), al señalar que los resultados de las investigaciones en el campo afectivo pueden variar por factores como el estatus económico, la etnicidad, factores propios de la institución escolar o el propio docente, lo que resalta la importancia del contexto en que se aprende matemática y de los entornos económicos y socioculturales a los que pertenezcan los estudiantes.

Los resultados por zona de ubicación del colegio indican la existencia de diferencias en la variable "actitud hacia la matemática" y en la variable "percepción de la actitud hacia la matemática del padre", levemente favorables en promedio para los colegios de las zonas rurales. El hallazgo es concordante con el obtenido por Agüero, Meza y Suárez (2017) para la variable autoconfianza matemática e igualmente sorprendente porque es bien conocido que estas instituciones enfrentan condiciones desfavorables para la enseñanza de la matemática en comparación con los de las zonas urbanas (Meza, Agüero y Calderón, 2013). No se encontraron diferencias por tipo de colegio en la variable "percepción de la actitud hacia la matemática de la madre".

Se encontraron relaciones significativas, positivas y moderadas entre las variables "actitud hacia la matemática", "percepción de la actitud hacia la matemática de la madre" y "percepción de la actitud hacia la matemática del padre". Estos productos coinciden con los hallazgos de Sanabria (2016) y Leonhardes (2017), quienes además, encontraron 
una relación significativa y moderada entre las dos últimas, para muestras de estudiantes costarricenses más pequeñas. Como elemento para la práctica de estos hallazgos se deriva la necesidad de que los colegios desarrollen actividades de motivación a los padres y madres de familia sobre la importancia, pertinencia, utilidad y belleza de la matemática.

\section{CONCLUSIONES}

La investigación permite llegar a las siguientes conclusiones:

a. Aproximadamente, un $81,1 \%$, un $83,5 \%$ y un $78 \%$ de los estudiantes muestran niveles de actitud hacia la matemática, percepción de la actitud hacia la matemática de la madre y percepción de la actitud hacia la matemática del padre entre alto y moderado, respectivamente.

b. No existen diferencias en el nivel de actitud la matemática entre hombres y mujeres.

c. Hay diferencias en el nivel de percepción de la actitud hacia la matemática de la madre entre hombres y mujeres, con niveles mayores en promedio para los hombres.

d. Se dan diferencias en el nivel de percepción de la actitud hacia la matemática del padre entre hombres y mujeres; vale resaltar que los hombres muestran, en promedio, niveles mayores.

e. No existen diferencias en el nivel de percepción de la actitud hacia la matemática de la madre entre hombres y mujeres, según la zona de ubicación del colegio.

f. Se mantienen diferencias en el nivel de actitud hacia la matemática según la zona de ubicación del colegio, con media superior para los estudiantes de los colegios de la zona rural.

g. Existen diferencias en el nivel de percepción de la actitud hacia la matemática del padre entre hombres y mujeres según la zona de ubicación del colegio, con nivel promedio mayor para los estudiantes de la zona rural.

h. Prevalece una relación significativa, positiva y moderada entre las variables actitud hacia la matemática y percepción de la actitud hacia la matemática de la madre.

i. Hay una relación significativa, positiva y moderada entre las variables actitud hacia la matemática y percepción de la actitud hacia la matemática del padre.

j. Existe relación significativa, positiva y moderada entre las variables percepción de la actitud hacia la matemática de la madre y percepción de la actitud hacia la matemática del padre.

\section{AGRADECIMIENTOS}

Se agradece el apoyo de la Vicerrectoría de Investigación y Extensión del Instituto Tecnológico de Costa Rica y también a los colegios participantes.

\section{REFERENCIAS}

Agüero, E., Meza, G. ySuárez, Z. (2017). Attitude toward usefulness of mathematics of Costa Rican high school students. Modern Journal of Language Teaching Methods (MJLTM), 7 (8), 162-168.

Akay, H. y Boz, N. (2010). The effect of problem posing oriented analyses-II course on the attitudes toward mathematics and mathematics self-efficacy of elementary prospective mathematics teachers. Australian Journal of Teacher Education, 35 (1), 1-75. Recuperado de http://files.eric.ed.gov/ fulltext/Ej908190.pdf.

Aparicio, A. y Bazán, J. L. (2005). Actitud y rendimiento en Estadística en profesores peruanos. Acta Latinoamericana de Matemática Educativa, 19, 644-650. Recuperado de http://www.ime.usp. br/ jbazan/download/ALME19.pdf.

Auzmendi, E. (1992). Las actitudes hacia la matemática/estadística en las enseñanzas media y universitaria. España: Mensajero.

Banks, J. (1964). Learning and teaching arithmetic. Boston: Allyn \& Bacon.

Besharat, M. (2003). Parental perfectionism and children's test anxiety. Psychological reports, 93 (3), 1049-1055. 
Berenguel, E., Gil, F., Montoro, A. B. y Moreno, M. (2015). Influencia de la autoconfianza y el perfil motivacional en el "flujo" en matemáticas. En C. Fernández, M. Molina \& N. Planas (Eds.). Investigación en Educación Matemática (XIX, pp. 173-181). Alicante: SEIEM. Recuperado de http:// rua.ua.es/dspace/bitstream/10045/51386/1/2015Actas-XIX-SEIEM_15.pdf.

Burga, A. (2006). La unidimensionalidad de un instrumento de medición: perspectiva factorial. Revista de Psicología de la PUCP, XXIV (1), 53-80.

Carmona, J. (2004). Una revisión de las evidencias de fiabilidad y validez de los cuestionarios de actitudes y ansiedad hacia la estadística. Statistics Education Research Journal, 3 (1), 5-28.

Cea, M. (1999). Metodología cuantitativa: estrategias y técnicas de investigación social. Madrid, España: Síntesis.

Fennema, E. y Sherman, J. A. (1976). FennemaSherman mathematics attitude scales. Instruments designed to measure attitudes toward the learning of mathematics by males and females. Journal for Research in Mathematics Education, 7 (5), 324326.

Gairín, J. (1990). Las actitudes en educación. Un estudio sobre la educación matemática. Barcelona: Boixareu Universitaria.

Gil, J. (1999). Actitudes hacia la estadística. Incidencia de las variables sexo y formación previa. Revista Española de Pedagogía, 214, 567-590.

Gil, N., Blanco, L. y Guerrero, B. (2006). El papel de la afectividad en la resolución de problemas matemáticos. Revista de educación, (340), 551-569. Recuperado de: http://dialnet.uniroja.es/servlet/ articulo? codigo $=2100483$.

Gómez-Chacón, I. (2000). Matemática emocional. Los afectos en el aprendizaje matemático. Madrid: Narcea S.A.

Gómez-Chacón, I. (2010). Actitudes de los estudiantes en el aprendizaje de la matemática con tecnología. Enseñanza de las ciencias, 28 (2), 227-244.
González-Pienda, J.A., Núñez, J.C., Álvarez, L., Roces, C., González-Pumariega, S., González, P., Muñiz, R., Valle, A., González-Cabanach, R., Rodríguez, S. y Bernardo, A. (2003). Adaptabilidad y cohesión familiar, implicación parental en conductas autorregulatorias, autoconcepto del estudiante y rendimiento académico. Psicothema 15: 471-477.

Jiménez, K. y Montero, E. (2013). Aplicación del modelo de Rasch, en el análisis psicométrico de una prueba de diagnóstico en matemática. Revista digital Matemática, Educación e Internet, 13 (1), 1-23

Leonhardes, E. (2017). Estudio sobre "percepción de la actitud de la madre hacia la matemática" y "percepción de la actitud del padre hacia la matemática" y su relación con "la expectativa de éxito en matemática" de las y los estudiantes de tres colegios públicos de la provincia de Cartago (Tesis de licenciatura, Instituto Tecnológico de Costa Rica), Costa Rica.

Lozano, L. y De la Fuente-Solana, E. (2009). Diseño y validación de cuestionarios. En Pantoja-Vallejo (coord.), Manual básico para la realización de tesinas, tesis y trabajos de investigación (pp. 251274). España: Editorial EOS.

Magalhães, A. (2007). Ansiedade face aos Testes, Género e Rendimento Académico: um estudo no Ensino Básico (Tese de mestrado, Universidade do Minho, Portugal), Portugal.

Martínez, O. (2008). Actitudes hacia la matemática. Sapiens, 9 (1), 237-256. Recuperado de http:// www.redalyc.org/articulo.oa?id=41011135012.

Mato, D. (2006). Diseño y validación de dos cuestionarios para evaluar las actitudes y la ansiedad hacia las matemáticas en alumnos de educación secundaria obligatoria (Tesis doctoral). Universidad de la Coruña, España. Recuperada de: http:// hdl.handle.net/2183/12688.

Mato, M., Muñoz, J. y Chao, R. (2014). Influencia de la profesión de los padres en la ansiedad hacia la matemática y su relación con el rendimiento 
académico en alumnos de secundaria. Ciencias Psicológicas, 8 (1), 69-77.

McLeod, D.B. (1989). Beliefs, attitudes and emotions: new views of affect in mathematics education. En D.B. McLeod y V.M. Adams (Eds.), Affect and Mathematical Problem Solving: A New Perspective (pp. 245-258). New York: Springer-Verlang.

Meza, L. G., Agüero, E. y Calderón, M. (2013). La teoría en la práctica educativa: una perspectiva desde la experiencia de docentes graduados/as de la carrera "Enseñanza de la matemática asistida por computadora". Revista digital Matemática, Educación e Internet, 13 (1), 1-24.

Meza, L. G. y Azofeifa, R. (2009). Actitud hacia la matemática de las y los estudiantes del curso Matemática General en el ITCR. Recuperado de: http://hdl.handle.net/2238/6278.

Michigan Department of Education. (2001). What research says about parent involvement in children's education in relation to academic achievement. Recuperado en Julio de 2015, del sitio Web del gobierno de Michigan: https://www. michigan.gov/documents/Final_Parent_Involvement_Fact_Sheet_14732_7.pdf.

Montero, Y. H., Pedroza, M. E., Astiz, M. S. y Vilanova, S. L. (2015). Caracterización de las actitudes de estudiantes universitarios de Matemática hacia los métodos numéricos. Revista Electrónica de Investigación Educativa, 17 (1), 88-99. Recuperado de http://redie.uabc.mx/vol17no1/contenidomontero-etal.html.

Morales, P. (2008). Estadística aplicada a las Ciencias Sociales. Madrid: Universidad Pontificia Comillas.

Nortes, R. y Nortes, A. (2014). ¿Tienen ansiedad hacia las matemáticas los futuros matemáticos? Profesorado. Revista de currículo y formación del profesorado, 18(2), 153-170. Recuperado de http:// www.redalyc.org/articulo.oa?id=56732350009.

Peleg-Popko, O. (2002). Children's Test Anxiety and Family Interaction Patterns. Anxiety, Stress \& Coping, 15 (1): 45-59.
Pérez-Tyteca, P. (2012). La ansiedad matemática como centro de un modelo causal predictivo de la elección de carreras (Tesis doctoral). Universidad de Granada, España.

Pérez-Tyteca, P., Castro, E., Rico, L. y Castro, E. (2011). Ansiedad matemática, género y ramas de conocimiento en alumnos universitarios. Enseñanza de las Ciencias, 29 (2), 237-250.

Quiles, M. N. (1993). Actitudes hacia las matemáticas y el rendimiento escolar. Comunicación, Lenguaje y Educación, 18, 115-124.

Ripoll, J. (2011). La d de Cohen como tamaño del efecto. Recuperado de: http://clbe.wordpress. com/2011/10/26/la-d-de-cohen-como-tamanodel-efecto.

Romagnoli, C. y Cortese, I. (2015). ¿Cómo la familia influye en el aprendizaje y rendimiento escolar? Ficha VALORAS actualizada de la $1^{\text {a }}$ edición "Factores de la familia que afectan los rendimientos académicos"(2007). Disponible en Centro de Recursos VALORAS: www.valoras.uc.cl.

Sanabria, J. (2016). Estudio de los niveles de "actitud hacia la matemática", "percepción de la actitud del padre hacia la matemática" y "percepción de la actitud de la madre hacia la matemática" que manifiestan los estudiantes de los colegios Francisca Carrasco Jiménez, San Luis Gonzaga y Experimental Bilingüe José Figueres Ferrer en el año 2015 (Tesis de licenciatura, Instituto Tecnológico de Costa Rica, Costa Rica). Costa Rica.

Torío, S., Peña, J.V. e Inda, M. (2008). Estilos de educación familiar. Psicothema, 20 (1): 62-70.

Ursini, S. (2014). Afectos y diferencias de género en estudiantes de secundaria de bajo desempeño en matemáticas. Educación Matemática [en línea]. Recuperado de http://www.redalyc.org/articulo. oa?id=40540854013 > ISSN 1665-5826. 